\title{
RADIATION PRESSURE IN NOVA EJECTA
}

James W. Younger

Department of Astronomy

Indiana University

The main features of the spectral evolution of novae consist of: 1. An overall increase in absorption line velocities and emission line widths as a function of time past maximum light. 2. The subsequent appearance of an absorption line system at roughly twice the velocity of the previous absorption line system. 3. Some absorption line systems apparently consist of multiple absorption components at different velocities.

In light of these features a method is presented to investigate the possible effects of radiation pressure due to optically thick lines within the ejecta of a nova. The high flow velocities (500 to $1500 \mathrm{~km} / \mathrm{sec}$ ) compared with the mean thermal velocities (10 km/sec) suggest that the gross features of the radiation field will be determined by the macroscopic flow field. The radiative transfer in the lines can then be treated by the Sobolev or Escape Probability Method as described by Rybicki and Humer (1978). The radiation pressure computed by this method is then coupled to a one-dimensional fluld dynamics computer code in order to investigate any possible dynamical effects and observable consequences in the line profile shapes.

\section{REFERENCES}

Rybicki, G.B., and Hummer, D.G. 1978, Ap.J., 219, 654. 